\title{
Using Rare Breeds in Animal-Assisted Activities: A New Model Proposed at the "Animal Farm" in Ladispoli (Rome, Italy)
}

\author{
Antonio Pizzuti Piccoli ${ }^{1} \&$ Agnieszka Lidia Kaczmarczyk $^{1}$ \\ ${ }^{1}$ Natura per Tutti Onlus Organization, Ladispoli (Rome), Italy \\ Correspondence: Antonio Pizzuti Piccoli, Natura per Tutti Onlus Organization, Via Monteroni 1265, Ladispoli \\ (Rome) 00055, Italy. Tel: 39-069-920-6063. E-mail: info@fattoriapertutti.it
}

Received: September 11, 2016

Accepted: October 12, 2016

Online Published: November 15, 2016

doi:10.5539/jas.v8n12p27

URL: http://dx.doi.org/10.5539/jas.v8n12p27

\begin{abstract}
In Europe many indigenous local breeds of domestic animals are at risk of extinction; the European Union supports multi-functionality activities (as the growth of Social Farms) in agriculture, also with the aim of save the biodiversity. In Italy, Social Farms are still in a pioneering phase; there are no pre-established models and the different realities of social farming, in recent years, have tried to put together some good practices referring to a personal field experience rather than coded models. The Animal Farm, social farm in Ladispoli (Rome, Italy), has developed a functional model, which is proposed in the present work, which consists in realizing together reproduction of rare breeds, educational activities and social farm activities. The innovative idea is to employ rare domestic animals in rehabilitative activities (AAA animal-assisted activities), encouraging their dissemination and preservation. The workshops are organized in daily activities where the various institutions of the territory (rehabilitation centers for disables, hospitals, etc.) bring their users (individual assets or groups) to carry out animal-assisted activities and practical workshops. The processed path type is aimed at people with medium and severe mental disabilities. The workshop is structured in two levels of activity: routine works and creative work. The experiments implemented within the AAA paths in the Animal Farm of Ladispoli (Rome, Italy) confirm the key role of animals as a facilitator in rehabilitation programs for the disabled. The Animal Farm proposes a model, repeatable in other realities, that allows the realization of animal-assisted activities with rare domestic breeds, preserving them from extinction. It is reported a preliminary study about the positive effects of using endangered races for AAA laboratories.
\end{abstract}

Keywords: animal-assisted activities, good practice model, rare breeds, rehabilitative workshop, social farm

\section{Introduction}

In Europe, many indigenous local breeds of domestic animals are at risk of extinction (Grunenfelder, 2001; Bigi \& Zanon, 2008). The European Union encourages the conservation measures of the domestic varieties, also stimulating the growth of multi-functionality activities in agriculture, which includes all activities that increase the social role of the agricultural sector (Di Iacovo \& O'Connor, 2009).

Educational Farms are recent realities in the multi-functionality evolution of farm in Italy, developed especially in the last two decade; these are farms where people can visit and learn about domestic animals and breeding cycles, getting in touch with the rural world now so far from the daily activities of most of the population, accustomed to urban contexts (Sofo \& Natile, 2013).

In addition to the Educational Farm in recent years, taking the experiences already developed in the US and in the Nordic countries of Europe, begin to appear on the Italian scene the Social Farms, farms where one of the main activities, if not all, is conducting therapeutic rehabilitation activities aimed at people with disabilities; often disable people are included in the production process (Hassink \& Van Dijk, 2006; Van Elsen, Herz, Ehlers, Schafer, \& Merckens, 2014).

Today are recognized by the international medical community some therapeutic activity employing pets as facilitators to achieve results in the field of rehabilitation; in particular we are talking about Animal-Assisted Activities AAA and Animal-Assisted Therapy AAT. (Katcher \& Beck, 1983; Alessandrini et al., 1998)

In Italy, Social Farms are still in a pioneering phase; only in 2015 the Italian government has drafted a national law on social farming (still without application). There are no pre-established models and the different realities 
of social farming, in recent years, have tried to put together some good practices referring to a personal field experience rather than coded models (Finuola \& Pascale, 2008; ARSIAL, 2011).

The Animal Farm, social farm in Ladispoli (Rome, Italy), is an agricultural center, realized on 2 ha of surface, in which are raised domestic animals belonging to rare breeds in danger of extinction. In particular are raised mammals as Sardinian donkey, Ragusano donkey, Sopravissana sheep, Girgentana goat, Little Aries rabbit, Leprino rabbit of Viterbo; several breeds of birds are raised: Ancona chicken, Paduan chicken, Sicilian Buttercup chicken, Leghorn chicken, pigeons Florentine pigeon, Modena's Triganino pigeon and Reggianino pigeon. The farm activities, other than raising, are based on two main actions: environmental educational activities for schools (in the last years 6000 students and visitors per year on average) and rehabilitation workshop dedicated to people with disabilities (Animal-Assisted activities).

The Animal Farm has developed a functional model, which is proposed in the present work, which consists in realizing together reproduction of rare breeds, educational activities and social farm activities. The innovative idea is to employ rare domestic animals in rehabilitative activities, encouraging their dissemination and preservation in Italy and neighboring countries. During the activities from May 2014 to June 2016 were collected preliminary data on the positive effects of using endangered races for AAA laboratories.

\section{Materials and Methods}

Animal Farm is based on an extensive type of farming model. Each group of animals has the possibility of moving free in a large enclosure, or go free grazing, and has a shelter for night and periods of bad weather.

Each race is bred with one or more reproductive groups; each unit consists of one male and three or four females for breeding (with the exception of monogamous species such as pigeons).

Breeding takes place, for races with short reproductive cycle (birds, rabbits, guinea pigs) only in the spring, to be able to have individuals sub adults in September. For races with longer reproductive cycle expects the complete weaning of born to give them. Every year, a small part of animals is used to renew breeding.

All the structures present were made by the disabled participants in several practical workshops over the years.

Using rare breeds in the AAT and AAA activities contributes to their preservation and dissemination. In fact one of the main causes of extinction of domestic races is their low use for human activities. Many varieties have not more productive value (for example the sheep Sopravissana) and are no longer used in the working activities (for example the Donkey Ragusano); the result is their numerical reduction with the risk of permanent disappearing (FAO, 2010; Scherf \& Pilling, 2015). Their re-employment in rehabilitation therapies and activities may contribute to the increase of these varieties and may restore the numerical strength of the breed beyond the edge of survival values. In this case they also provide many sub-products that benefit society.

The Animal Farm, since 2001, brings to the development of a good practice model, proposed in this work, which can be exported and implemented in other situations; the farm staff consists of agronomists, psychotherapists, ethologists, farmers who work together as a team in the implementation of educational and rehabilitation activities.

In Animal Farm are considered Animal-Assisted activities all actions related to the interaction between man and animal; in particular come up to the AAA daily activities of animal care (feeding, cleaning of housing, etc.), but also particular exercises planned in specific workshops (schemes of work with donkeys and other animals).

The workshops are organized in daily activities where the various institutions of the territory (rehabilitation centers for disables, hospitals, etc.) bring their users (individual assets or groups) to carry out animal-assisted activities and practical workshops. The processed path type is aimed at people with medium and severe mental disabilities.

Activities are conducted, at the beginning, always with the presence, as well as of the operators of farm, by operators of rehabilitation services who daily work with the involved disabled people. This is a facilitation giving security in an initially completely new environment for the involved disabled. Over time the role of the farmer operator becomes increasingly dominant as they increase the personal knowledge and familiarity for the spaces of the farm.

The proposed workshops aim at recovery of psycho-physical well-being of those who participate, to promote a sense of responsibility and autonomy and an opportunity to socialize. During practical workshops the participants can decide the time and space needed to perform the required tasks (for example to check if there is enough eat in the mangers, fill empty ones with adequate feed for the species of the raised animals), developing a sense of autonomy and the ability to make decisions. 
It tries to encourage users to provide suggestions regarding the execution of the works, in particular those concerning the establishment of structures for the farm. The decisions do not come from the top, but it involves them in the choice of materials, the order of execution of work, operational strategies. Initially participants are monitored and guided in practical activities by operators and are gradually encouraged to independently perform some stages of the farm management. Undoubted is the importance, in the rehabilitation phase, of operational independence and of the awareness of their abilities, that the subject can successively express in new and different work environments. The workshop is structured in two levels of activity: routine works and creative work.

\subsection{Routine Works}

In this first level are included all care and handling of farm animals (Figure 1). The routine work is used to put the participant in confidence with the places and facilities of the farm, giving him a sense of security, and promoting his self-reliance. The activities proposed in routine are structured such as to be realized in the daily time of permanence of the person in the farm; routine jobs such as feeding the animals or cleaning the corrals, must be structured to be able to finish within the time of two or maximum three hours and give the perception of the finite work. This promotes a sense of gratification for a job started and completed in the defined time frame.

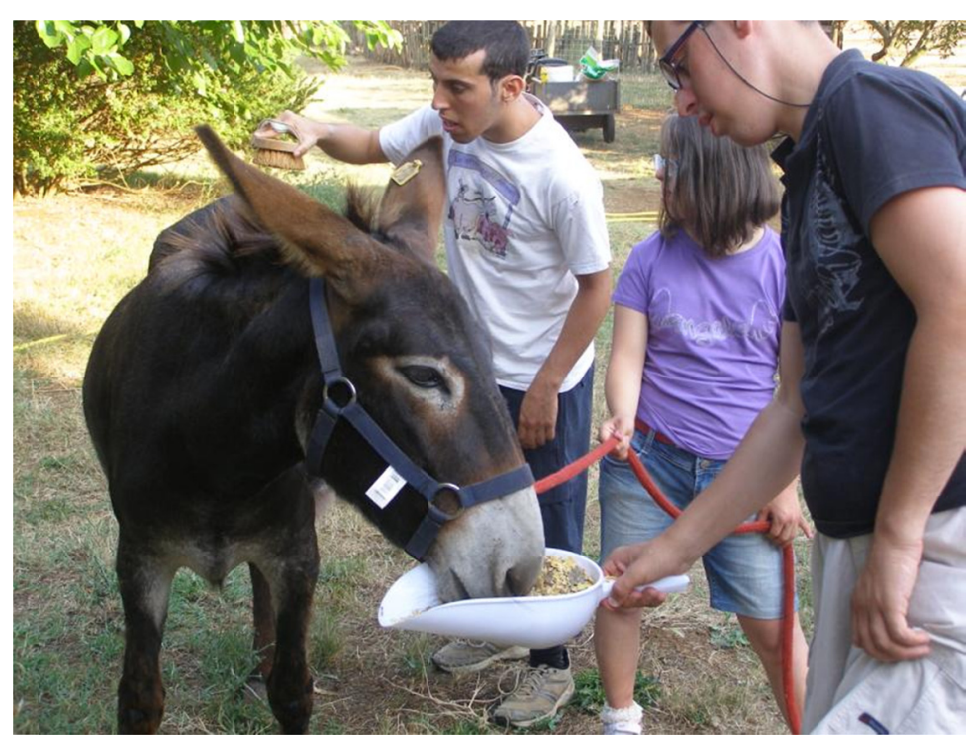

Figure 1. Participants in routine work, taking care of a donkey

\subsection{Creative Work}

The second operating level involves the participants to the construction of facilities for the farm (fences, greenhouses, feeders, etc), first conceived and designed, then built together; the activities will end in several days of work, in every day they are applied to build and terminate a single part of the structure. This level gives the idea of something finite, realized in all its phases of construction by the participants, persons who work will have contributed to improving the capabilities of the farm; this gives a sense of belonging and increases self-esteem.

The working day is marked by three moments:

$>$ Preparation of participants and initial meeting with the definition of assets and assignment of duties and organization of the times;

$>$ Working activities, interrupted by a break for a snack;

$>$ Final meeting of the day with feedback comments.

In the period between May 2014 and June 2016, weekly, were asked to participants in workshops, during the phase of initial meetings, simple questions about which animals they wanted to take care, the choice was always between two date options, including ordinary farm animals (industrial breeds of chicken, rabbits, etc.) and rare breeds (Ancona, Paduan and Sicilian Buttercup chicken, Little Aries rabbit, etc.). 
During the observation period there have been involved 73 different people and the reactions of the participants about rare breeds and not used in AAA laboratories, were collected.

As part of the daily workshops they are inserted obstacle factors that contribute to maintaining the functions of the person involved and to increase motor and psychic abilities. For example the animal fences have a lock and to the person involved is given a set of keys, from which to choose the right one; the input of the fences is hampered by small barriers (about $20 \mathrm{~cm}$ high) that require to the person to lengthen the stride, doing a movement exercise that otherwise would not be daily done.

Of course all the activities proposed are carried out safely and in accordance with hygienic standards corresponding to the parameters of the law currently in force.

\section{Results}

At the end of the breeding season, thanks to the work carried out in the AAA laboratories, the farm has animals in excess which are entrusted to people who want to establish small breeding groups for familiar use. In this way, in addition to keep animals to employ them in therapeutic programs, the farm spreads indigenous breeds in small familiar farms, encouraging their conservation.

In Table 1 it is shown the implementation costs and the annual cost of maintenance of the farm. The farm was founded in 2001 and within six years has achieved financial autonomy, regaining even the initial funding. The farm cost of maintaining derived by contributions, by tickets of schools and visitors for educational activities and by the contribution of the participants in rehabilitation workshops.

During the observation period, between May 2014 and June 2016, 73 participants in workshops have been involved in data collecting; approximately $83 \%$ of participant have chosen to work with breeds at risk of extinction, were also cataloged the reactions and behaviors with both typology of proposed animals.

Table 1. Costs of implementation and management of a farm based on the Animal Farm model

\begin{tabular}{ll}
\hline Initial cost of realization of a social farm & $46,000.00 €$ \\
Annual cost of management & $106,000.00 €$ \\
Total cost of realization and management for the first year & $152,000.00 €$ \\
\hline
\end{tabular}

Note. Costs are in euro referred to Italy.

In 14 years of activity (2001-2015), the Animal Farm has carried environmental education activities dedicated to native breeds in danger of extinction for a number of 30000 people (of which about $70 \%$ school-age children). On average about 60 disabled persons participate yearly to the workshops of animal-assisted activities. More than 3000 animals were bred and free distributed throughout the country $(78 \%$ of these domestic birds, the remaining mammals).

The Animal Farm, over the years, has established itself as a good practice model repeatable in other rural areas of the planet.

\section{Discussion}

The experiments implemented within the AAA paths in the Animal Farm of Ladispoli (Rome, Italy) confirm the key role of animals as a facilitator in rehabilitation programs for the disabled (Pugliese, 2005).

According to other authors (Melson, 2003; Megan, Souter, \& Miller, 2007; Pichot, 2012) the condition of disability implies that others take care of disabled persons; in farming activities such condition is reversed: are people with disabilities or other form of disadvantage who take care of other organisms. In addition, animals react to human care and reward with events that could be called "affection". There is a sort of positive feedback that helps to strengthen the sense of individual responsibility and consolidates self-esteem. People with disabilities find a further increase in self-esteem participating to a conservation path of everyone's heritage as are the domestic breeds at risk of extinction.

Not secondary is the fact that working in a farm, to manage and treat animals and structures, allows to stay outdoors, with very wide possibilities of movement, favoring the physical activity and decreasing the sense of constriction given by the closed spaces. To carry out activities related to animal care multiple motor function are necessary: they are, in fact, required unilateral movements, bilateral (both arms or both legs), bimanual movements (one hand performs the action and the other helps), bending, stretching, rhythmic or/and automatic movements, the 
coordination of movements and global purposes, the hand-eye coordination, muscular strength and endurance, the learning of the strength dosage, the maintenance for a certain period of time of the upright position.

The animals have been shown to provide significant benefits to the cardiovascular and neuro-muscular apparatus. The activities that are realized in the structure can urge all human senses; sight, touch, hearing, smell are stimulated at various stages of the breeding process. It is sensory stimuli that can be particularly useful in the rehabilitation of people with medical conditions that generate high closing by the external environment (Darrah, 1996; Sams, Fortney, \& Wilenbring, 2006). All these experiences are then used as a springboard for improvements in the sphere of human relations. In subjects who have socialization problems or difficulties the animal assumes the role of mediator, favoring the relationship with other people (Bardill \& Hutchinson, 1997; Martin \& Farnum, 2002; Taylor, Fraser, \& Signal, 2016).

These benefits for human people have ben registered in all the social farms that are today present in Europe and in the American continent (Hassink \& Van Dijk, 2006). However, analyzing the work done, there are very few realities that turn their attention to the use of animals in danger of extinction and choose them on an exclusive basis for its activities; with the only exception for donkeys, now rare in the entire European continent (Scherf \& Pilling, 2015).

From observations of the behavior and reactions, during the practical workshops, some important factors have emerged; the heterogeneous color of different rare breeds poultry, compared to homogeneous color of industrial breeds, enhancing the creativity and stimulate the participants. Some animals inspire safety in participants, such as the Paduan hen, with his tuft of feathers on the head, that has reduced visibility and is therefore less mobile; this chicken is favored by the participants because it has less sudden movements and reduces their stress factors. The same thing is observed for the Florentine pigeon, a pigeon of medium large size, that prefers walking instead fling. Docility of Little Aries rabbit, during keeping activities, increases the agreement of participants. The stress reduction due to less unpredictability of animal movements during interaction, and increasing the pleasure of interacting with the preferred animals, are the facilitators mechanisms for the success of the AAA activities.

Over the years experience has led to indicate the domestic breeds that are better employable in AAA activities. First of all the medium-small breed of donkeys, Sardinian and Ragusano breed; the donkey is used in specific activities of onotherapy (Milonis, 2010) or in simple actions of government and occurrence are very usefully also rare breed of sheep (Sopravissana sheep) and goat (Girgentana goat).

A very important role have little farm animals, (including the chicken breeds Ancona, Paduan, Sicilian Buttercup, Leghorn and the pigeons Florentine, Modena's Triganino and Reggianino); the daily care and maintenance of poultry houses and dovecotes is an easily spendable activity in AAA laboratories organization. Among others the reproduction of chicks with the incubator is an important moment and very emotionally engaging in daily life of the farm. Among the smaller animals also include domestic rabbits (race Little Aries rabbit and Leprino rabbit of Viterbo) who take the real connotations of the Pet, and can be used in real activities of "Pet Therapy".

These preliminary information confirm and encourage the undertaken work, although it will be necessary further studies in the future for more confirmations. In conclusion, the proposed model, repeatable in other realities, allows the undoubted value of promoting the realization of animal-assisted activities that rare domestic breeds, preserving them from extinction.

\section{Acknowledgements}

The Authors are grateful to the CARICIV Foundation and Nuove Frontiere Organization for the support given to the "Animal Farm" of Ladispoli.

\section{References}

Alessandrini, B., Braccili, T., Del Papa, S., Di Blasio, M., Montebello, D., Pediconi, O., \& Scaioli, S. (1998). AAT for children with disabilities and other problems. International Conference of Human-Animal Interaction "The changing role of animals in society", Praga.

ARSIAL (Agenzia Regionale per lo Sviluppo e l'Innovazione dell'Agricoltura del Lazio). (2011). Guida alle aziende di agricoltura Sociale del Lazio. Regione Lazio.

Bardill, N., \& Hutchinson, S. (1997). Animal-assisted therapy with hospitalized adolescents. Journal of Child and Adolescent Psychiatric Nursing, 10(1), 17-24. http://dx.doi.org/10.1111/j.1744-6171.1997.tb00208.x

Bigi, D., \& Zanon, A. (2008). Atlante delle razze autoctone. Edagricole.

Darrah, J. P. (1996). A pilot survey of animal-facilitated therapy in Southern California and South Dakota nursing home. Occupational Therapy International, 3(2), 105-121. http://dx.doi.org/10.1002/oti.31 
Di Iacovo, F., \& O’Connor, D. (2009). Supporting Policies for Social Farming in Europe. Progressing Multi-functionality in Responsive Rural Areas. ARSIA, Firenze.

FAO. (2010). Breeding strategies for sustainable management of animal genetic resources. FAO Animal Production and Health Guidelines, No. 3. Rome.

FAO. (2015). The second report on the state of the world.

Finuola, A., \& Pascale, A. (2008). L'agricoltura sociale nelle politiche pubbliche. Istituto Nazionale di Economia Agraria.

Grunenfelder, H. P. (2001). Risorse genetiche agrarie in Italia. Monitoring Institute for Rare Breeds and Seed in Europe.

Hassink, J., \& Van Dijk, M. (2006). Farming for Health: Green-Care Farming Across Europe and the United States of America. Springer. http://dx.doi.org/10.1007/1-4020-4541-7

Katcher, A. H., \& Beck, A. M. (1983). New perspectives on our lives with companion animals. University of Pennsylvania Press.

Martin, F., \& Farnum, J. (2002). Animal-assisted therapy for children with pervasive developmental disorders. Western Journal of Nursing Research, 26(6), 657-670. http://dx.doi.org/10.1177/019394502320555403

Megan, A., Souter, M. A., \& Miller, M. D. (2007). Do Animal-Assisted Activities Effectively Treat Depression? A Meta-Analysis. Anthrozoös, 20(2).

Melson, G. F. (2003). Child development and the human companion animal bond. American Behavioral Scientist, 47(1), 31-39. http://dx.doi.org/10.1177/0002764203255210

Milonis, E. (2010). Io e gli asini-Attività di mediazione con gli asini. Fausto Lupetti Editore

Pichot, T. (2012). Animal-Assisted Brief Therapy-A solution focused approach. Taylor and Francis Group New York.

Pugliese, A. (2005). Pet therapy-Strategie di intervento e linee guida. Armando Siciliano Editore.

Sams, M. J., Fortney, E. V., \& Willenbring, S. (2006). Occupational therapy incorporating animals for children with autism: A pilot investigation. American Occupational Therapy Association, 60, $268-274$. http://dx.doi.org/10.5014/ajot.60.3.268

Scherf, B. D., \& Pilling, D. (2015). The Second Report on the State of the World's Animal Genetic Resources for Food and Agriculture. FAO Commission on Genetic Resources for Food and Agriculture Assessments, Rome. Retrieved from http://www.fao.org/3/a-i4787e/index.html

Sofo, A., \& Natile, M. C. (2013). La realtà rurale delle fattorie didattiche in Italia. Lulu Editore.

Taylor, N., Fraser, H., \& Signal, T. (2016). Animal-Assisted Therapies and Ethical Considerations: A Programme Example from Central Queensland, Australia. British Journal of Social Work, 46(1), $135-152$. http://dx.doi.org/10.1093/bjsw/bcu115

Van Elsen, T., Herz, G., Ehlers, H., Schafer, W., \& Merckens, K. (2014). INCLUFAR - Inclusive Farming: A New Educational Approach in Social Farming. Proceedings of the 4th ISOFAR Scientific Conference 'Building Organic Bridges' at the Organic World Congress 2014, Istanbul, Turkey.

\section{Copyrights}

Copyright for this article is retained by the author(s), with first publication rights granted to the journal.

This is an open-access article distributed under the terms and conditions of the Creative Commons Attribution license (http://creativecommons.org/licenses/by/4.0/). 